\title{
Experimental Investigation of Aerodynamic Hysteresis Using a Five-Degree-of-Freedom Wind-Tunnel Maneuver Rig
}

\author{
Z. Gong* \\ Nanjing University of Aeronautics and Astronautics, Nanjing, People's Republic of China \\ S. Araujo-Estrada, $₫$ M. H. Lowenberg,, \pm and S. A. Neild $\stackrel{\S}{-}$ \\ University of Bristol, Bristol, England BS8 1TR, United Kingdom \\ and \\ M. G. Goman- \\ De Montfort University, Leicester, England LE1 9BH, United Kingdom \\ DOI: $10.2514 / 1 . C 034995$
}

\begin{abstract}
The high-incidence aerodynamics of a lightweight jet trainer aircraft model has been investigated using a novel five-degree-of-freedom (DOF) dynamic maneuver rig, recently updated with improved actuation and data acquisition systems, in the $7 \times 5 \mathrm{ft}$ closed-section low-speed wind tunnel at the University of Bristol. The major focus was to identify the nonlinear and unsteady aerodynamic characteristics specific to the stall region and which affect free-to-move aircraft-model behavior. First, the unstable equilibrium states in the limit-cycle regions were stabilized, and so observed, over a wide range of angles of attack using a simple elevator feedback control law based on pitch angle and pitch-rate sensor measurements. Tests with two DOF, namely, the aircraft model and rig-arm pitch angles, revealed the existence of static hysteresis in the normal force acting on the aircraft model in the stall region. Unlocking the aircraft model in roll and yaw accompanied by feedback stabilization of the lateraldirectional modes of motion demonstrated the onset of asymmetric aerodynamic rolling and yawing moments in this four-DOF configuration. This observation implicitly indicates a link between the static hystereses in the normal aerodynamic force with an onset of aerodynamic asymmetry. The experimental results show the efficiency of the updated multi-DOF actively controlled maneuver rig in providing insight into complicated aerodynamic effects within the stall region.
\end{abstract}

\begin{tabular}{|c|c|c|}
\hline \multicolumn{3}{|r|}{ Nomenclature } \\
\hline$C_{L}$ & $=$ & aircraft-model lift coefficient \\
\hline$C_{L_{c}}^{L}$ & $=$ & rig-arm compensator lift coefficient \\
\hline$C_{l}$ & $=$ & aircraft-model rolling-moment coefficient \\
\hline$C_{l_{\beta}}$ & $=$ & $\begin{array}{l}\text { derivative of } C_{l} \text { with respect to sideslip angle (effective } \\
\text { dihedral) }\end{array}$ \\
\hline$C_{l_{\delta_{a}}}$ & $=$ & derivative of $C_{l}$ with respect to aileron angle \\
\hline$C_{N}$ & $=$ & aircraft-model normal force coefficient \\
\hline$C_{\mathrm{Nc}}$ & $=$ & compensator normal force coefficient \\
\hline$C_{\mathrm{Nc}_{\delta_{\mathrm{ec}}}}$ & $=$ & $\begin{array}{l}\text { derivative of } C_{\mathrm{Nc}} \text { with respect to compensator elevator } \\
\text { angle }\end{array}$ \\
\hline$C_{\mathrm{Nc}_{\theta_{g}}}$ & $=$ & derivative of $C_{\mathrm{Nc}}$ with respect to rig-arm pitch angle \\
\hline & $=$ & aircraft-model yawing-moment coefficient \\
\hline$C_{n_{\beta}}$ & $=$ & derivative of $C_{n}$ with respect to sideslip angle \\
\hline$C_{n_{\delta_{r}}}$ & $=$ & derivative of $C_{n}$ with respect to rudder angle \\
\hline$k_{p}$ & $=$ & aircraft-model roll-rate gain \\
\hline$k_{q}$ & $=$ & aircraft-model pitch-rate gain \\
\hline$k_{q_{a}}$ & $=$ & rig-arm pitch-rate gain \\
\hline$k_{r}$ & $=$ & aircraft-model yaw-rate gain \\
\hline
\end{tabular}

Received 10 March 2018; revision received 15 October 2018; accepted for publication 16 October 2018; published online Open Access XX epubMonth XXXX. Copyright (C) 2018 by the American Institute of Aeronautics and Astronautics, Inc. All rights reserved. All requests for copying and permission to reprint should be submitted to CCC at www.copyright.com; employ the ISSN 0021-8669 (print) or 1533-3868 (online) to initiate your request. See also AIAA Rights and Permissions www.aiaa.org/randp.

*Lecturer, Department of Aerospace Engineering, College of Aerospace Engineering.

Tesearch Associate, Department of Aerospace Engineering, Queens Building, University Walk.

${ }^{\ddagger}$ Professor of Flight Dynamics, Department of Aerospace Engineering, Queens Building, University Walk. Senior Member AIAA.

${ }^{\S}$ Professor in Nonlinear Structural Dynamics, Department of Mechanical Engineering, Queens Building, University Walk.

"Professor of Dynamics, Faculty of Technology, School of Engineering and Sustainable Development, The Gateway. Senior Member AIAA. compensator, and aircraft model from vertical strut gimbal center in horizontal direction when $\theta_{a}=0$

aircraft-model lift arm measured from vertical strut gimbal

location of the center of gravity of the rig arm, compensator, and aircraft model from vertical strut gimbal center in vertical direction when $\theta_{a}=0$

$=$ aircraft-model angle of attack

$=$ aircraft-model sideslip angle aircraft-model aileron angle aircraft-model aileron demand input aircraft-model elevator angle compensator elevator angle compensator elevator demand input aircraft-model elevator demand input aircraft-model rudder angle aircraft-model rudder demand input aircraft-model pitch angle rig-arm pitch angle aircraft-model roll angle aircraft-model trim roll angle aircraft-model yaw angle 


\section{Introduction}

$\mathbf{T}$ HE deterioration of aerodynamic characteristics at high angles of attack due to separated flow conditions results in various types of loss of control and dynamic instability. These can limit the maneuverability for fighter aircraft and affect the flight safety of transport airplanes.

Wind-tunnel testing techniques are traditionally used for characterization of aerodynamic loads for aircraft design, aerodynamic modeling, and flight simulation. Various wind-tunnel rigs are used for measuring aerodynamic forces and moments in static, forced-oscillation, and rotary balance tests. The aerodynamic data obtained at high angles of attack are less reliable than at low-tomoderate incidence due to the high sensitivity of separated flow to wind-tunnel test conditions, such as the Reynolds number, flow turbulence, level of aeroelastic vibrations, and imperfection of windtunnel flow and aircraft-model geometry. Data obtained in different wind tunnels for the same aircraft model may differ significantly under high-angle-of-attack conditions [1]. Research is needed to understand the complex flow phenomena due to flow separation in the stall region within the flight envelope, and, as part of this, the use of novel experimental testing and the related aerodynamic modeling techniques must be developed. Many unsteady and nonlinear aerodynamic effects due to flow separation can be spoiled or even totally missed due to inappropriate test conditions or aerodynamic data processing.

Flight tests with large-scale controllable aircraft models dropped from a helicopter or airplane were effectively used in the past to complement wind-tunnel tests in evaluating critical flight regimes, such as stall and spin [2]. Recently, there has been an increase in activity to develop experimental techniques for conducting virtual flight (or direct physical flight simulation) of an aircraft model in a wind-tunnel working section. This approach is aimed at investigating the stability and control characteristics of the model with a view to extracting the aerodynamic data [3-7]. The idea is to allow an aircraft model to fly in a wind-tunnel working section with partially constrained motion imposed by a supporting system. The aircraft model has a number of degrees of freedom (DOF), and this is expected to ensure more realistic aerodynamic loads than in captive (static and forced oscillation) testing, due to more natural model motion generated by deflections of control surfaces.

These techniques also potentially allow a significant reduction in aeroelastic coupling between an aircraft model and its support system compared with traditional dynamic testing. In static, forced oscillation, and rotary balance tests, aircraft models are usually mounted on long cantilever stings through a strain gauge balance; such support systems are prone to sting-balance-model vibrations, which are especially strong under stall conditions due to the coupling between unsteady separated flow and system elastic eigenmodes [을] . The excited vibrations of the sting-balance-model system have a considerable intrusive effect on the separated flow structure, changing the measured aerodynamic loads [10]. A suitable virtual flight dynamic rig can be used to provide model motion measurements in different configurations with varying number of DOF, with reduced interaction with support structure dynamics (due to much lower eigenfrequencies relative to conventional rigs), facilitating a deeper insight into complicated unsteady and nonlinear aerodynamics strongly affected by separated flow conditions.

This paper presents the experimental results obtained on the recently updated five-DOF dynamic maneuver rig, developed at the University of Bristol [11-15], and now with improved actuation and data acquisition instrumentation. The tests were conducted at the University of Bristol $7 \times 5 \mathrm{ft}(2.1 \times 1.5 \mathrm{~m})$ closed-section low-speed wind tunnel. A specially developed high-speed wireless message collection-distribution network allowed the distributed motion parameters to be recorded with synchronized time stamps in microseconds for phase-sensitive analysis; a low command-response loop latency (below $10 \mathrm{~ms}$ ) made possible the implementation of realtime closed-loop feedback control strategies. Exploiting this new control capability, and specifically the feedback stabilization, this paper reports on experiments that examine the aircraft model's nonlinear and unsteady aerodynamic characteristics, specifically for stall conditions. This is achieved through the use of feedforward and feedback control strategies across a range of different combinations of the maneuver rig DOF. The results provide a significant new insight into the cause of nonlinear limit-cycle behavior observed in previous test campaigns with the maneuver rig.

The updated maneuver rig and the aircraft-model systems are briefly presented in Sec. II. The characteristics of poststall selfsustained pitch oscillations of the aircraft model and their active control stabilization are detailed in Sec. III. The results of tests with one DOF (model pitch) and two DOF (model pitch and rig-arm pitch) are presented in Secs. IV and $\underline{\mathrm{V}}$, respectively. And, finally, Sec. VI outlines the results from tests with the maneuver rig in a four-D $\overline{\mathrm{OF}}$ configuration with longitudinal and lateral-directional modes.

\section{Maneuver Rig and Model Control System}

The maneuver wind-tunnel rig used in this study is shown schematically in Fig. 1a. An aircraft model is mounted on the arched end of the supporting sting on a three-DOF gimbal giving the aircraft model freedom to move in pitch, yaw, and roll within the following limits: $|\theta| \leq 20 \mathrm{deg},|\psi| \leq \infty$, and $|\phi| \leq 42 \mathrm{deg}$, but diminishing to $\pm 15 \mathrm{deg}$ as $\theta$ reaches its minimum or maximum values. The opposite, downstream end of the sting is equipped with an aerodynamic compensator formed from four fins with deflectable trailing-edge control surfaces. The sting, or rig arm, is mounted to a vertical support strut via a three-DOF gimbal. Deflections of the rig arm in pitch $\theta_{a}$ and yaw $\psi_{a}$ induce aircraft-model movement in heave (up/down) and sway (left/right), respectively. (Note that these motions are not precisely pure heave and sway, as the aircraft is constrained to move in an arc.) Essentially, the aircraft model itself has five DOF because of the lack of a surge (fore/aft) DOF.

The first version of the maneuver rig was designed with a two-DOF gimbal for the aircraft model $[11,12]$ but, aiming for the simulation of asymmetrical departure at high angles of attack, the aircraft model had recently been equipped with a three-DOF gimbal [15]. This allows the simulation of rapid lateral departures. To avoid the aircraftmodel gimbal impacting its mechanical constraint during deflection in roll, the rig arm may be rotated to follow the aircraft-model roll motions; this is achieved using feedback control driving the aerodynamic compensator on the rear of the rig arm [15].

The rig system is designed to be configured with different DOF by locking or releasing each axis or DOF independently. The control surfaces on the four fins of the aerodynamic compensator mounted at the rear of the rig arm are primarily used for compensation of aerodynamic, inertial, and gravitational forces generated on the rig arm, but also can be used for forcing motion of the aircraft model in heave and sway.

The aircraft model used in this work, shown in Fig. 1b, is the same as in previous studies using the maneuver rig [12-15], namely, an approximate replica of a one-sixteenth-scale B $\overline{\mathrm{Ae}} \overline{\mathrm{Hawk}}$ jet trainer aircraft model. The ailerons, elevator, and rudder are all actuated, and can be used to control the aircraft model either manually via a stick input or by using a command and stability augmentation control system via a ground-station computer. (The model actually has an allmoving horizontal tail, but it is referred to in this paper as an elevator.)

In addition to changes in the mechanical design of the maneuver rig [15], recent enhancements include equipping the aircraft model and compensator surfaces with embedded microprogrammed control units for both sensing and actuating. To minimize mechanical friction from electrical wiring, the remote nodes use XBee Wi-Fi modules [16] for wireless communication with the ground-station computer (or PC node). A distributed message delivery system runs on a realtime operating system in a $1 \mathrm{kHz}$ task loop on each remote node, which synchronizes the local clock with the PC node by a simplified version of the Network Time Protocol [17]. A health-monitoring program running on the $\mathrm{PC}$ shows that the clock offsets are within $200 \mu \mathrm{s}$ and the time latency of the command-response loops is kept below $10 \mathrm{~ms}$. These upgrades provide two important features, namely, the ability to postprocess records from distributed nodes with 


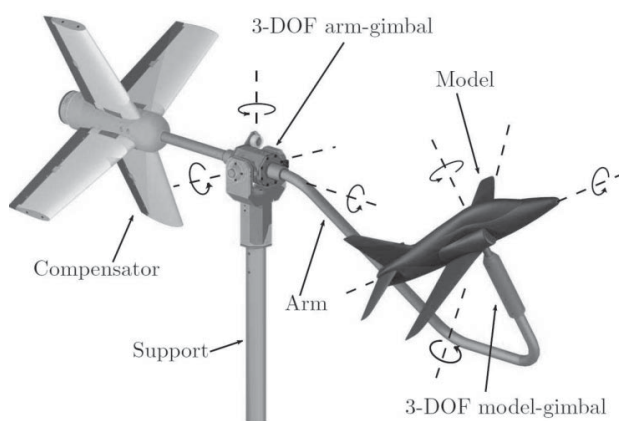

a) Schematic diagram of rig

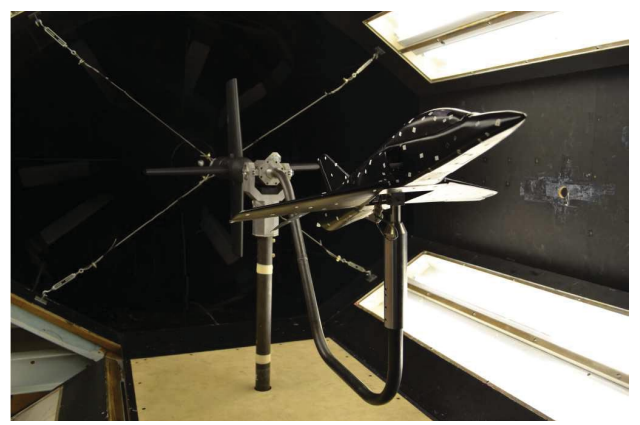

b) Aircraft pitch-only configuration in $7^{\prime} \times 5^{\prime}$ closed section wind tunnel

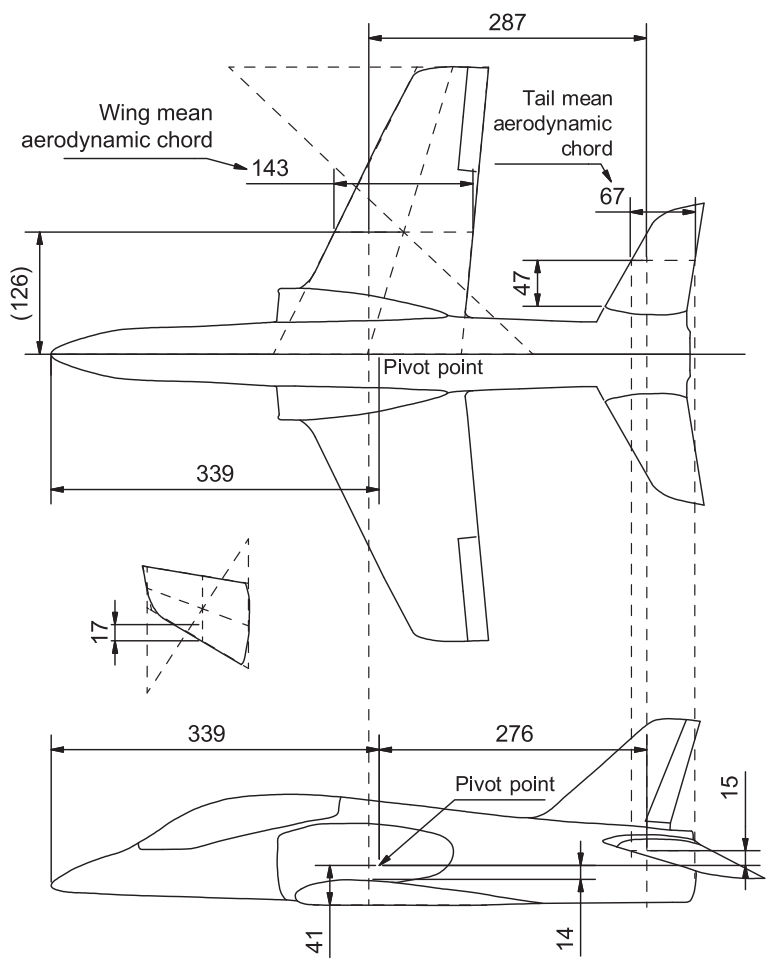

c) Model geometry (dimensions in $\mathbf{m m}$ )

8 Fig. 1 Maneuver rig and aircraft model.

microsecond-accuracy time stamps and to implement feedback control with a communication delay of less than $10 \mathrm{~ms}$.

The data acquisition system has also been upgraded by improving the method by which the attitude of the aircraft model is observed. In the previous maneuver rig design, the encoders and potentiometers on the model and rig gimbals were used to measure the relative attitudes of aircraft model-to-arm and arm-to-wind-tunnel flow. From these signals, the absolute attitudes and c.g. position of the aircraft model were calculated. However, this setup is unable to account for any aeroelastic deformation of the arm. Comparative tests showed that the maximum measurement errors caused by the aeroelastic effects can exceed $1 \mathrm{deg}$ in the aircraft-model attitude. The lead/lag errors in phase angle are also significant. In the upgraded rig, the kinematic measurement has been supplemented by an inertial measurement unit located inside the aircraft model, allowing a Kalman filter to be used to estimate the aircraft attitude relative to the wind-tunnel flow while also correcting the gyro drift [18].

The servo-control loop design for the control surface deflections on both the aircraft model and the compensator has been improved in terms of both the response rate and accuracy. A tuned proportionalplus-derivative control law, based on the experimentally identified linearized motor model, forms the linear part of the servo-control loop. In addition to this, a nonlinear inversion control law is used to minimize steady-state errors by compensating the hinge friction via a Coulomb-like friction model. The servo-control code was implemented in the real-time operating system to sample angles and actuate motors. The digital signal processor engines in the microprogrammed control units have been used to implement a Butterworth filter and numerical difference calculations for angular rates. As a result, the servo deflection may be viewed as an approximately linear second-order component, modeled by a second-order transfer function with the natural frequency of $30 \mathrm{rad} / \mathrm{s}$ and damping ratio 0.8 . The rate saturation for the aircraft-model actuators is approximately $300 \mathrm{deg} / \mathrm{s}$. The closed-loop transfer function indicates that, with the servo controller, the lag in response to input commands is reduced to approximately $100 \mathrm{~ms}$.

\section{Poststall Pitch Oscillations and Their Active Control Suppression}

It was reported in a number of previous publications, for example in [13], that this Hawk aircraft model exhibits self-excited limit-cycle oscillations (LCOs) in pitch motion when trimmed by the elevator to angles of attack in the poststall region. Those studies were not able to provide convincing evidence of the flow mechanisms responsible for the onset of this phenomenon in the trimmed state. The development of LCOs, due to the instability of the aircraft model, significantly transforms the separated flow and aerodynamic loads: as a result, it is impossible to observe flow over the model and to measure aerodynamic loads in steady conditions with a constant angle of 
attack. The active control approach allows the aircraft model to be stabilized, eliminating the onset of large-amplitude LCOs.

The maneuver rig in these tests was configured to allow aircraft pitch-only motion (i.e., a one-DOF setup).

Figure 2a shows the variation of pitch angle $\theta(t)$ of the aircraft model, obtained in the wind-tunnel test using the upgraded maneuver rig. A slowly changing ramplike elevator input was applied over a time interval of $450 \mathrm{~s}$. During the model pitch-up motion, the LCOs were excited at $\delta_{e}=-7 \mathrm{deg}$ and disappeared at $\delta_{e}=-21 \mathrm{deg}$ via a transition to a stable equilibrium state. One should note that the model equilibrium states are agitated due to the wind-tunnel flow turbulence and aeroelastic vibrations of the rig arm. The trimmed states of the aircraft model have small-amplitude stochastic oscillations around the equilibrium point. The averaged LCOs for a number of different elevator deflections are shown in Fig. $2 b$ in the form of phase trajectories.

After reaching the maximum pitch angle $\theta=26.5 \mathrm{deg}$, the elevator reversed, generating a pitch-down motion. During the slow decrease in the model pitch angle, the stable equilibrium states existed until approximately $\delta_{e}=-16 \mathrm{deg}$. At this elevator deflection, the LCOs were excited, and there was an abrupt increase in the response amplitude. The levels of oscillation after this transition are the same as in the LCOs observed in the pitch-up motion. With further pitch-down elevator deflection, the LCOs disappeared after a smooth decrease of the amplitude to zero at

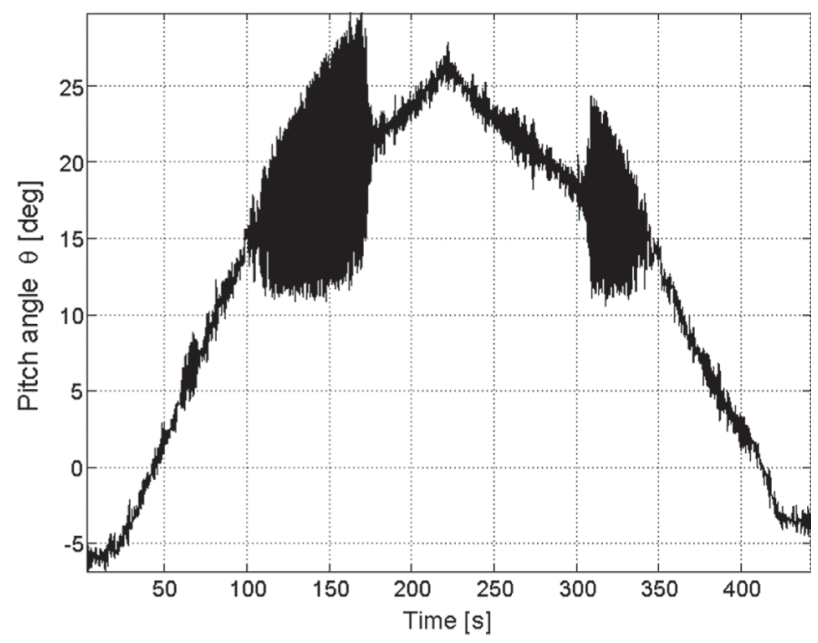

a) Experimental record of the pitch angle variation vs elevator deflection

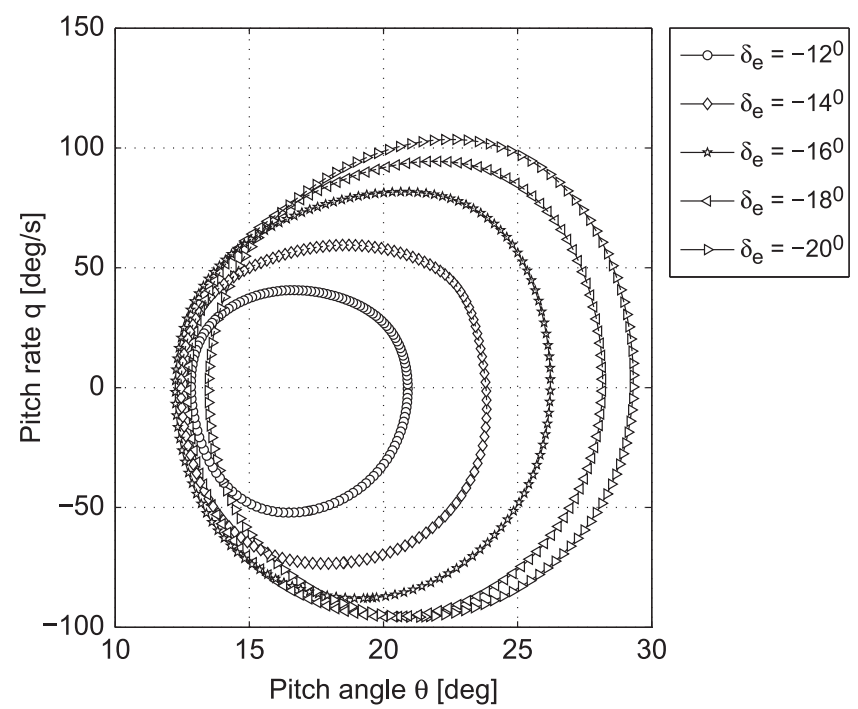

b) Open loop LCOs at several elevator deflections

Fig. 2 a) Measured response to slow pitch-up and -down elevator inputs, and b) open-loop LCOs. $\delta_{e}=-7 \mathrm{deg}$ : this is the same condition as for the LCO onset in the pitch-up motion.

Figure $3 \mathrm{a}$ is a sketch of the bifurcation structure for equilibrium states (solid lines) and LCO amplitude (square markers) vs elevator deflection; the transitions between different steady-state branches of the aircraft-model behavior are indicated by the vertical arrows. These steady-state solutions (solid lines for equilibria and square markers for LCOs) were experimentally observed in the wind tunnel (see Fig. 2a). The sketch in Fig. 3a also includes provisional unstable steady states for equilibria, shown as the dashed line segment between $\delta_{e}=-7 \mathrm{deg}$ and $\delta_{e}=-16 \mathrm{deg}$. From topological considerations, one should expect that this segment of the equilibrium states has oscillatory instability. Figure 3 a also shows the provisional amplitudes for unstable LCOs, marked by hollow circles. Being topologically consistent, the presented bifurcation diagram in Fig. 3a explains the onset and structure of the hysteretic transitions between the LCOs and stable equilibrium states.

The elevator of the Hawk model has sufficient control power to suppress the LCOs in the pitch motion of the aircraft model. This was demonstrated in previous studies with application of a stabilizing feedback control law using the maneuver rig with significantly higher time delays in the sensor-controller-actuator chain that is now achievable.

In the current study with updated maneuver rig hardware/software characteristics (see previous section), the feedback control law for elevator deflection is of the form:

$$
\delta_{e}=\delta_{e_{d}}(\alpha)+\frac{k_{\theta} s}{s+\omega} \theta+k_{q} q
$$

in which the values of $k_{q}, k_{\theta}, \omega$, and $\delta_{e_{d}}(\alpha)$ are given in the Appendix. Pitch-rate feedback was deployed to increase aircraft-model damping, while the pitch angle was fed back to augment static stability and improve rejection of external disturbances from windtunnel turbulence. The pitch angle $\theta(t)$ in Eq. (1) was obtained using the extended Kalman-filter-based observer, whereas the pitch rate $q(t)$ was taken directly from the inertial gyro measurement unit, and $\delta_{e_{d}}(t)$ is the elevator-angle demand.

The gain coefficients $k_{\theta}$ and $k_{q}$ in Eq. (1) were selected using iterative manual tuning on a linear mathematical model. This was constructed with aerodynamic characteristics estimated for the Hawk model at low angles of attack using steady-state solutions of the Reynolds-averaged Navier-Stokes [19] equations. This aerodynamic analysis, based on computational fluid dynamics simulations in Fluent [20], was carried out by Bai Yalei at the Nanjing University of Aeronautics and Astronautics in China.

The inclusion of the washout filter in Eq. (1) was to eliminate the influence of static offset in the pitch angle, and so maintain a steadystate elevator deflection equal to demand $\delta_{e}(\infty)=\delta_{e_{d}}$. Wind-tunnel tests demonstrated that the feedback control law (1) stabilizes the aircraft-model equilibrium states in the stall region, and at the same time eliminates the LCOs with low and large amplitudes at various elevator deflections $\delta_{e_{d}}$.

Figure $3 \mathrm{~b}$ shows the effect the controller (1) has on the aircraftmodel pitch angle $\theta(t)$ time history for the experimental setup. Initially, the feedback controller was turned off with the elevator deflection set to $\delta_{e}=-16 \mathrm{deg}$. At first, the aircraft-model response was oscillatory with increasing amplitude around the unstable equilibrium state until the oscillation becomes fully developed-this took about $2.5 \mathrm{~s}$. The period of the developed LCO was approximately $T=0.59 \mathrm{~s}$, and the peak-to-peak amplitude was around $\Delta \theta \approx 13 \mathrm{deg}$. The feedback controller (1) was switched on at $t=$ $468 \mathrm{~s}$ with the control demand set to $\delta_{e_{d}}=-16 \mathrm{deg}$. With the controller activated, the pitch-angle oscillations $\theta(t)$ converged within $2 \mathrm{~s}$ to the equilibrium state $\theta_{e}=18 \mathrm{deg}$ with an elevator deflection of $\delta_{e}=-16 \mathrm{deg}$. This equilibrium state was unstable in the open-loop system observed prior to $t=468 \mathrm{~s}$; the controller has successfully suppressed the LCO by stabilizing the equilibrium solution. In this equilibrium state, the elevator actively compensates for disturbances from the wind-tunnel turbulence and aeroelastic vibrations of the supporting rig arm, giving rise to slight fluctuations 


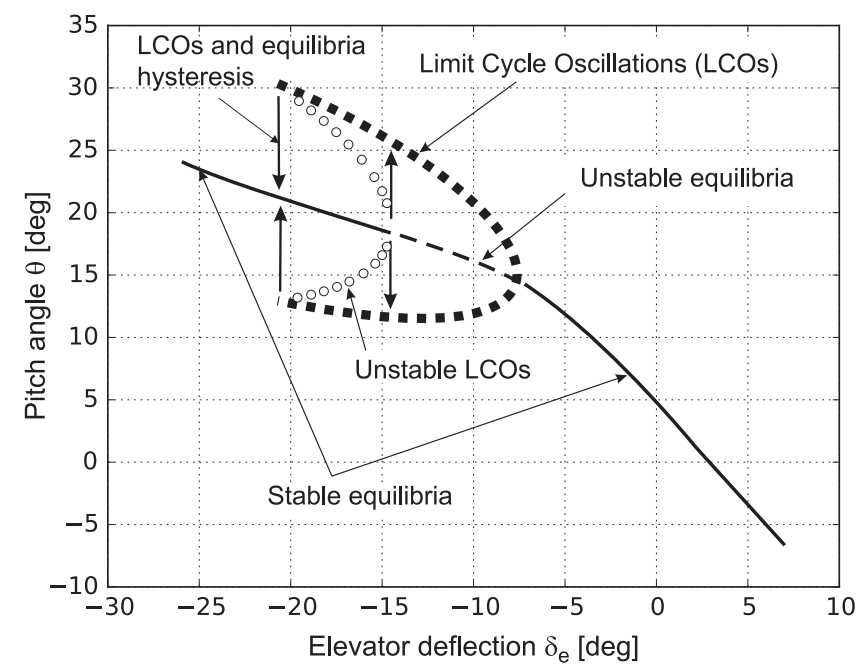

a) Sketch of bifurcation structure
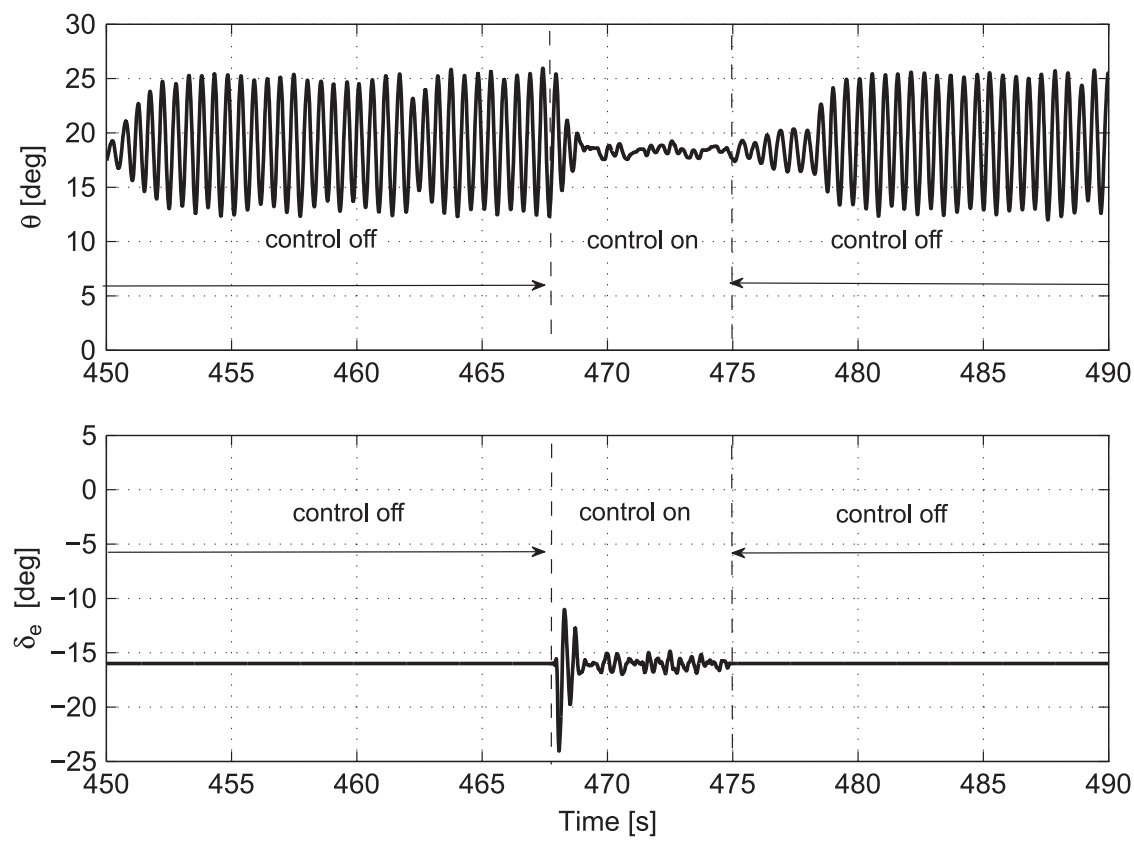

b) Active suppression of LCOs using feedback control

Fig. 3 a) Sketch of bifurcation diagram for equilibrium and LCOs, and b) suppression of the LCOs using controller [Eq. (1 $)$ ].

in $\delta_{e}$ about $-16 \mathrm{deg}$. The feedback control was switched off at $t=475 \mathrm{~s}$, and the aircraft-model LCO reappeared.

By testing at different command inputs over the range from $\delta_{e_{d}}=-5 \mathrm{deg}$ to $\delta_{e_{d}}=-25 \mathrm{deg}$, the controller (1) demonstrated its ability to suppress the large-amplitude LCOs and to stabilize the equilibrium branch with only marginal oscillations in the elevator deflection required to mitigate against external disturbances.

\section{Pitch-Only Tests with Feedback Control to Track Stabilized Equilibrium States}

Elimination of the large-amplitude LCOs and stabilization of the equilibrium states using the feedback controller (1) allow one to get an insight into possible equilibrium states, which are not observable in the aircraft model without stabilizing feedback elevator control. An investigation of the stabilized equilibrium states at different elevator deflections and their dependence on previous motion within the stall region is now possible in the closed-loop system.

Such tests, with the elevator demand $\delta_{e_{d}}$ in the stabilizing controller (1) set to a slowly varying ramp with $\dot{\delta}_{e_{d}}= \pm 0.2 \mathrm{deg} / \mathrm{s}$, were conducted on the rig configured for one DOF in pitch angle for the aircraft model. Figure $\underline{4}$ shows the time histories for the aircraft-model pitch angle $\theta(t)$ at slow pitch-up/pitch-down motions in the stall range of pitch angle $\theta \in[13,20 \mathrm{deg}]$. The loops have been repeated three times. The solid lines depict the ramp motions with increasing incidence, and the dotted lines depict the ramp motions with decreasing incidence. The stabilized pitch angles of the aircraft model in all the tests shown in Fig. 4a are quite noisy due to turbulence and rigarm vibrations. This noise blurs the subtle transitions in the pitch angle between different equilibrium branches. A Butterworth filter with a cutoff frequency of $0.2 \mathrm{~Hz}$ was used to reduce the effects of noise and smoothen the raw data for the model pitch angle $\theta(t)$. Each curve is processed twice-once forward and once backward in time-to retain zero-phase delay. After such filtering, the experimental curves became smoother, and the pitch-angle variations during elevator increase and decrease can be now distinguished from each other in Fig. 4b. The filtered experimental curves clearly show two zones of static hysteresis with transitions between different equilibrium branches. The first hysteresis loop is located in the range of pitch angle $\theta \approx 14-15 \mathrm{deg}$, and the second, wider, hysteresis loop is located over $\theta \approx 17-19 \mathrm{deg}$.

The equilibrium states provisionally shown in Fig. 3a can now be clarified. Figure $\underline{5}$ shows the stabilized equilibrium branches, observed 


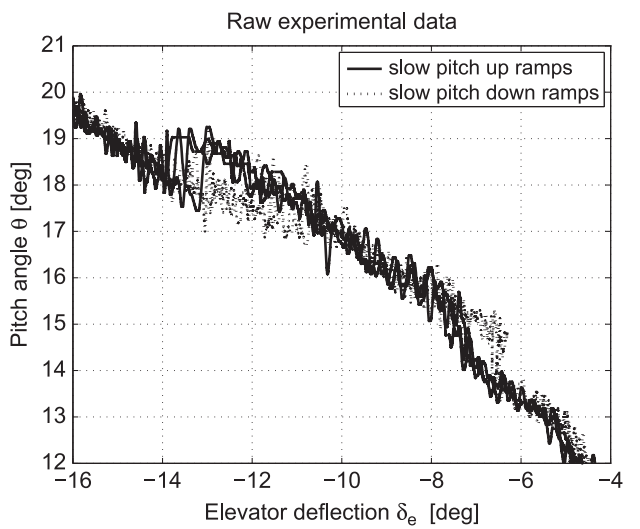

a) Experiment raw data

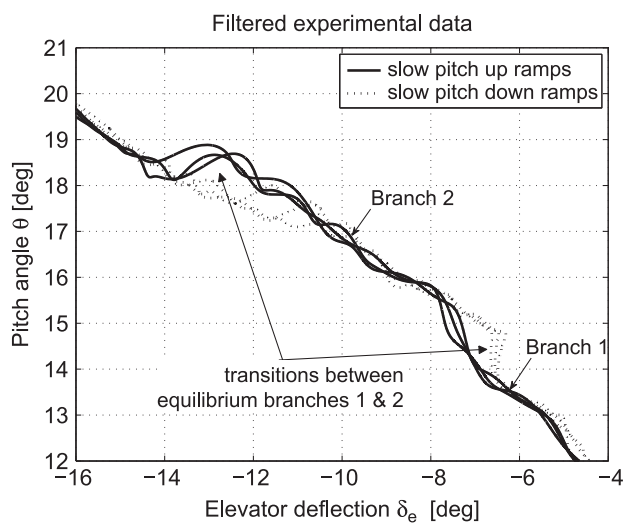

b) Experiment data after filtering

Fig. 4 Trimmed pitch angle $\theta$ for slow pitch-up and -down elevator deflections with stabilizing controller [Eq. (1)].

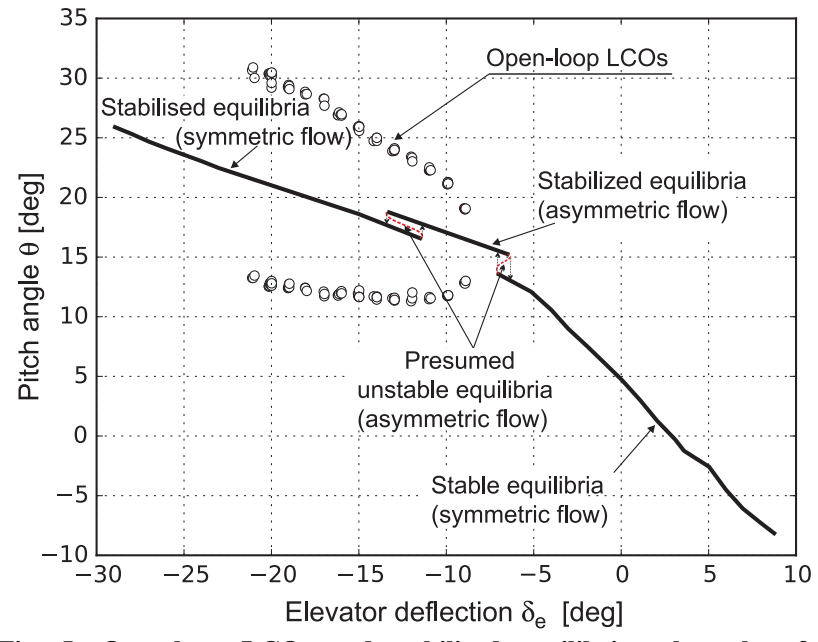

Fig. 5 Open-loop LCOs and stabilized equilibrium branches for aircraft-model pitch-only configuration.

experimentally using the actively controlled aircraft model, along with the amplitudes of LCOs observed in the open-loop experiments and presented in Fig. 3a. Nontypical aerodynamic responses with abrupt jumps can be effectively interpreted based on generic topological properties manifested in nonlinear dynamic systems. The two static hysteresis regions between the equilibrium branches in Fig. 5 indicate the bifurcational transitions between the equilibria solution branch at low and high $\alpha$ and a raised equilibrium branch in the range $\delta_{e}=-14$ to $-7 \mathrm{deg}$; this segment of equilibrium solutions is presumed to belong to the branches connected by fold bifurcations (with associated unstable equilibria connecting the segments).

The Hawk model in this test is only free in the longitudinal mode while the lateral-directional DOF are fixed. The bifurcational changes in pitch angle can be triggered by changes in the flow structure, which may be either symmetric or asymmetric in nature. Flow separation is highly sensitive to small geometric asymmetries of the Hawk model, and this makes the onset of asymmetric separated flow conditions more probable. The verification of such an assumption in this case is presented in Sec. VI.

The local angles of attack for the horizontal tailplane of the Hawk model remain low throughout the whole range of trim states considered, which ensures sufficient aerodynamic efficiency for active control stabilization of the unstable equilibrium solutions; it also supports the conclusion that all nonlinear aerodynamic effects are localized on the wing.

\section{Heave-and-Pitch Tests with Active Stabilization of Equilibrium States}

The test results shown in Fig. 5 demonstrate the nonlinear and bifurcational dependence of the pitch angle $\theta$ on the aircraft-model elevator deflection $\delta_{e}$, indicating the existence of singularities in the pitching-moment coefficient $C_{m}$. In the stall region, one can expect more pronounced nonlinearities in the aerodynamic force dependence.

The version of the maneuver rig used here does not allow direct measurements of the aerodynamic loads using a traditional strain gauge balance or load cell. However, its design allows the aerodynamic force on the aircraft model to be measured indirectly using the heave-and-pitch rig configuration. In this configuration, both the rig arm and the aircraft model are free to move in pitch with angles $\theta_{a}$ and $\theta$, respectively. The trim pitch angle of the aircraft model depends only on the aircraft-model elevator $\delta_{e}$, whereas the trim pitch angle for the rig arm depends on $\delta_{e}$ and the elevator deflection for the aerodynamic compensator $\delta_{\mathrm{ec}}$. Pitch-up and pitchdown ramp motions of the aircraft model were implemented using slow ramp inputs in aircraft elevator deflection $\delta_{e}$ similar to the tests presented in the previous section. In addition, the compensator elevators were used to stabilize the combined dynamics of the rig arm and the aircraft model to prevent the onset of the selfsustained LCOs.

With the rig arm free in pitch, it behaves as a mechanical balance scale. At every attitude of the aircraft model, the arm will find an equilibrium position when the aerodynamic force of the aircraft model will be compensated by the aerodynamic force acting on the compensator (see Fig. 6). A simplified moment balance equation, accounting for the aircraft-model aerodynamic forces (assumed to act at the aircraft gimbal center), compensator aerodynamic forces (assumed to act at the compensator quartermean-aerodynamic-chord position), and the gravitational force due to the combined model and rig, about the vertical supporting strut gimbal is given by

$$
\bar{q} S C_{N} \cdot X_{m}=\bar{q} S_{c} C_{\mathrm{Nc}} \cdot X_{c}+M g\left\{\cos \left(\theta_{a}\right) \cdot X_{\text {c.g. }}+\sin \left(\theta_{a}\right) \cdot Z_{\text {c.g. }}\right\}
$$

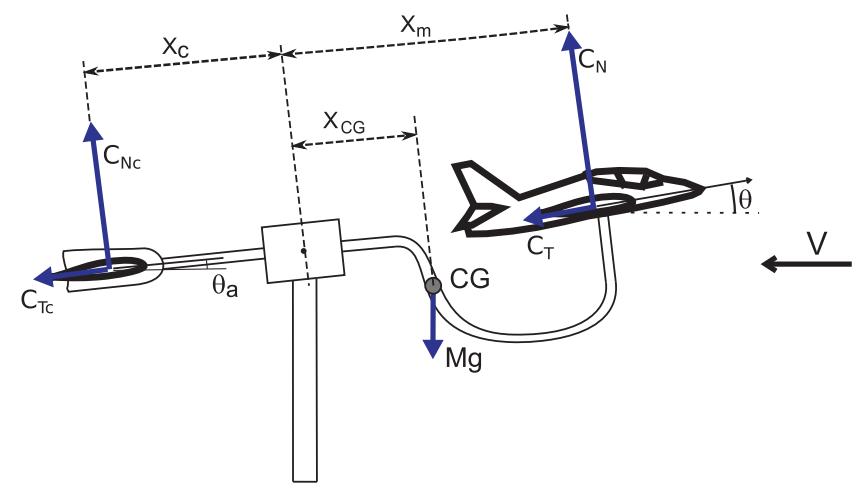

Fig. 6 Maneuver rig in heave-pitch two-DOF configuration. 
in which

$$
\begin{aligned}
C_{N} & =C_{L} \cos \left(\theta_{a}\right)+C_{D} \sin \left(\theta_{a}\right), \\
C_{\mathrm{Nc}} & =C_{L_{c}} \cos \left(\theta_{a}\right)+C_{D_{c}} \sin \left(\theta_{a}\right)
\end{aligned}
$$

and $C_{L}\left(\theta, \delta_{e}\right), C_{D}\left(\theta, \delta_{e}\right), X_{m}$, and $S_{m}$ are the aircraft-model aerodynamic lift and drag coefficients, normal-force moment arm to the vertical strut gimbal and wing area, respectively. Also, $C_{L_{c}}\left(\theta_{a}, \delta_{\mathrm{ec}}\right), C_{D_{c}}\left(\theta_{a}, \delta_{\mathrm{ec}}\right), X_{c}$, and $S_{c}$ are the compensator aerodynamic lift and drag coefficients, the normal-force moment arm, and the compensator aerodynamic area, respectively; $M$, $X_{\text {c.g. }}$, and $Z_{\text {c.g. }}$ are the effective mass and center-of-gravity location for the rig arm, compensator, and aircraft model combined.

The rig is configured (in terms of mass and c.g. location), such that the trim conditions are realized at low arm pitch angles $\theta_{a}$, where flow on the compensator is attached and the lift force $C_{L_{c}}$ can be presented as a linear function of the pitch angle $\theta_{a}$. Equation (2) can then be written:

$$
\begin{array}{r}
C_{N}\left(\theta, \delta_{e}\right)=\frac{S_{c} X_{c}}{S X_{m}}\left(C_{\mathrm{Nc}_{\theta_{a}}} \theta_{a}+C_{\mathrm{Nc}_{\delta_{\mathrm{ec}}}} \delta_{\mathrm{ec}}\right) \\
+\frac{M g\left\{\cos \left(\theta_{a}\right) \cdot X_{\text {c.g. }}+\sin \left(\theta_{a}\right) \cdot Z_{\text {c.g. }}\right\}}{\bar{q} S X_{m}}
\end{array}
$$

Using the small-angle approximations, $\cos \theta_{a}=1$ and $\sin \theta_{a}=\theta_{a}$, this becomes

$$
\begin{gathered}
C_{N}\left(\theta, \delta_{e}\right) \approx\left(\frac{S_{c} X_{c} C_{\mathrm{Nc}_{\theta_{a}}}}{S X_{m}}+\frac{M g Z_{\text {c.g. }}}{\bar{q} S X_{m}}\right) \theta_{a} \\
+\left(\frac{S_{c} X_{c} C_{\mathrm{Nc}_{\delta_{\mathrm{ec}}}}}{S X_{m}} \delta_{\mathrm{ec}}+\frac{M g X_{\text {c.g. }}}{\bar{q} S X_{m}}\right)
\end{gathered}
$$

Thus, the aerodynamic normal force coefficient $C_{N}$ can be considered to have a linear dependence on the arm pitch angle $\theta_{a}$.

In the tests with the heave-and-pitch rig configuration (Fig. 6), the aircraft model was trimmed and stabilized by the aircraft-model elevator $\delta_{e}$ using the feedback control law (1). The rig arm was controlled and stabilized using compensator elevator $\delta_{\mathrm{ec}}$ by feeding back the arm pitch-rate signal $q_{a}$ to increase the damping of the aircraft-model heave movement while retaining the same trimmed states:

$$
\begin{aligned}
\delta_{e} & =\delta_{e_{d}}+\frac{k_{\theta} s}{s+\omega} \theta+k_{q} q \\
\delta_{\mathrm{ec}} & =\delta_{\mathrm{ec}_{d}}+k_{q_{a}} q_{a}
\end{aligned}
$$

Figure 7a presents the experimental data for trim angles $\theta_{a}$ (the rigarm attitude) vs trim angles $\theta$ (the aircraft-model attitude). Note that, in trim conditions, the pitch angle equals the aircraft-model angle of attack $(\theta=\alpha)$. Two sets of slow pitch-up (solid lines) and slow pitchdown (dotted lines) ramp movements were conducted by varying the aircraft-model elevator demand $\delta_{e_{d}}(t)$ in a slow ramp while demanding zero compensator elevator deflection $\delta_{\mathrm{ec}_{d}}=0$.

The experimental data have been postprocessed to remove any high-frequency oscillatory component, arising from wind-tunnel turbulence, model vibrations, etc. Assuming a small angle of attack and no stall on the aerodynamic compensator, the aerodynamic force on the aircraft model is approximately proportional to the arm pitch angle $\theta_{a}$ [Eq. (4)], and so the curves shown in Fig. 7a can be viewed as representing the dependence of the aerodynamic force on the aircraft model on its pitch angle (or angle of attack). The aerodynamic normal force coefficient $C_{N}$ can be calibrated experimentally against the arm pitch angle $\theta_{a}$.

Considering in Fig. 7a the variation of rig-arm attitude $\theta_{a}$ as model pitch angle $\theta$ increases: after a nearly linear increase in $\theta_{a}$ with $\theta, \theta_{a}$

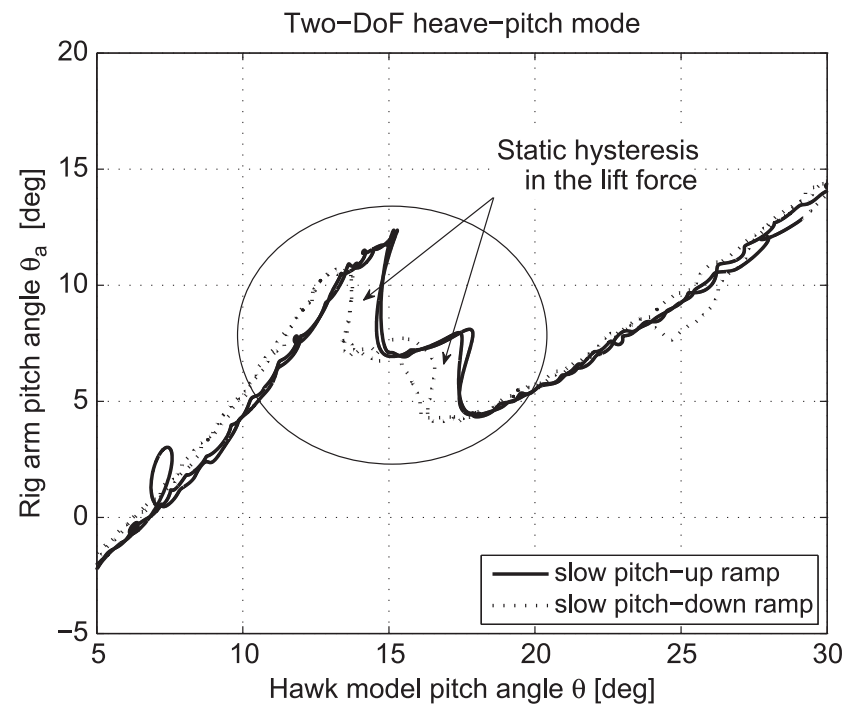

a) Experimental measurements after filtering

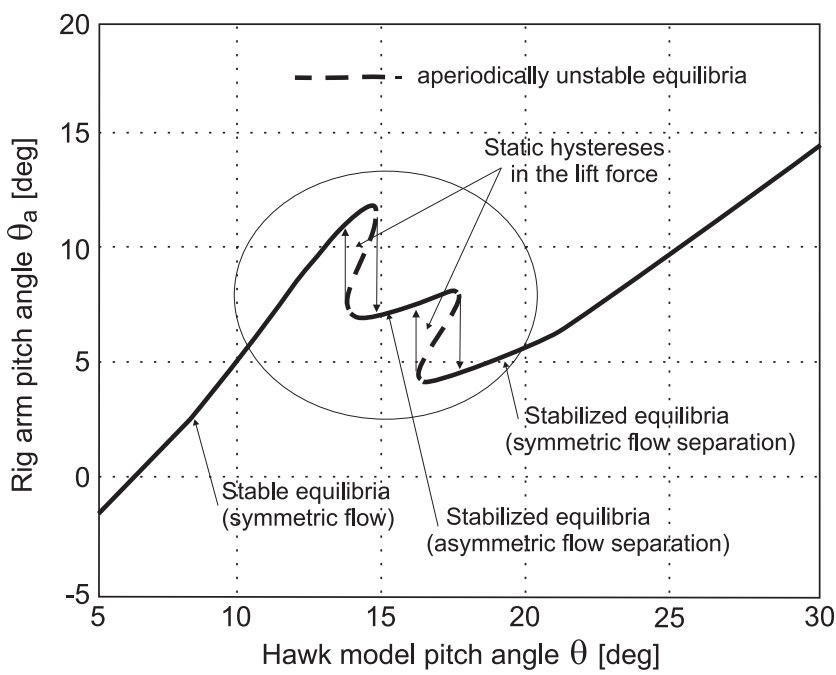

b) Presumed bifurcation diagram for $\theta_{\mathrm{a}} \mathrm{vs} \theta$

Fig. 7 Actively stabilized trim states $\theta_{a}$ and $\theta$ on the heave-pitch rig configuration (Fig. 6).

drops abruptly from 12 to $7 \mathrm{deg}$ at $\theta=15 \mathrm{deg}$. Note that the model pitch angle is equal to the angle of attack $(\alpha=\theta)$. A further slight increase in $\theta_{a}$ takes place until $\theta_{a}=8 \mathrm{deg}$ at $\theta=17.5 \mathrm{deg}$. At this point, a second abrupt drop in arm pitch angle occurs from $\theta_{a}=$ $8 \mathrm{deg}$ to $\theta_{a}=4 \mathrm{deg}$. A further increase in $\theta$ results in a further linear increase in $\theta_{a}$ until $\theta_{a}=14 \mathrm{deg}$ at $\theta=30 \mathrm{deg}$. During the slow reverse deflection of the aircraft-model elevator $\left(\dot{\delta}_{e}>0\right)$, there are again two vertical jumps, but now with increases in the rig-arm attitude. These both occur at roughly $1 \mathrm{deg}$ lower model pitch angle, or angle of attack, than the drops observed in the tests with increasing angle of attack. The abrupt transitions in the experimentally measured rig-arm attitude $\theta_{a}$ may be triggered by bifurcations in flow separation processes in the stall region. Because the rig-arm attitude relates directly to the lift-coefficient dependence on angle of attack $C_{L}(\alpha)$, these bifurcations in flow structure are reflected in the force coefficient in a similar way, namely, discontinuous jumps in the aerodynamic force acting on the aircraft model during slow changes in angle of attack $\alpha$. Similarly, the hysteretic variations in model trim angle of attack exhibited in Figs. $\underline{4}$ and $\underline{5}$ are linked to the hysteretic dependence of the pitching moment on angle of attack $C_{m}(\alpha)$.

In interpreting the sudden jumps in the experimental data, an analogy with bifurcational models is useful. Figure $7 \mathrm{~b}$ shows a hypothetical bifurcation diagram for stabilized equilibrium states $\theta_{a}$ and $\theta$. It includes three stable branches in $\theta_{a}$ dependence on pitch angle $\theta$ (recalling that, in trimmed conditions, $\theta=\alpha$ ). The 
intermediate branch, located in the range of $\theta \approx 14-17.5 \mathrm{deg}$, is identical to the intermediate branch identified earlier using one-DOF tests on the aircraft model, in which only the aircraft pitch angle was free to move (see Fig. 5). All three stabilized equilibrium branches are connected in the so-called saddle-node bifurcation points via hypothetically existing aperiodically unstable equilibrium branches (dashed lines). Abrupt jumps occur at these bifurcation points if the pitch angle is swept past them, forming the experimentally observed static hysteresis loops.

The cause of these abrupt transitions in the aircraft-model equilibrium states is connected with topological changes in the flow structure in the stall region. Transitions from attached to separated flow conditions and changes in structure of separated flow may be reflected in the aerodynamic loads in the form of jumplike changes. As discussed in Sec. IV, evidence suggests that the first transition toward the intermediate branch may correspond to the onset of asymmetric separated flow conditions, which exist in a narrow range of angles of attack. The second transition toward the third stabilized equilibrium branch is then assumed to be linked to symmetrization of the separated flow structure. These assumptions are examined further in the next section with the rig operating in four DOF (heave-pitchroll-yaw) to include lateral-directional effects.

\section{Four-DOF Coupled Longitudinal and Lateral-Directional Tests}

To improve the insight into the observed aerodynamic hysteresis of the aircraft model, tests were conducted with the aircraft gimbal unlocked in all three DOF (roll, pitch, and yaw) and the arm free to move in pitch to approximate aircraft heave motion. The aim was to identify whether lateral-directional aerodynamic moments are generated along with the bifurcational aerodynamic dependencies in the longitudinal dynamics. This rig configuration allows the aircraft model to move in roll and yaw DOF, facilitating the testing of possible onset of asymmetric aerodynamic moments. Many aircraft configurations at high angles of attack manifest the so-called in which feedback gains $k_{\phi}$ and $k_{\psi}$ augment aerodynamic stiffness in the roll and yaw deflections, respectively. The feedback gains $k_{\phi}$ and $k_{\psi}$ were selected with the objective of preventing rotation and limiting the trimmed angles in roll and yaw caused by asymmetric aerodynamic rolling and yawing moments. Feedback gains $k_{p}$ and $k_{r}$ are used to stabilize the lateral-directional modes and to supress wing-rock oscillations. Values adopted for these gains are presented in the Appendix.

To evaluate the aircraft-model roll and yaw angles at trim conditions, the following representations of the rolling and yawing aerodynamic moments are considered:

$$
\begin{aligned}
C_{l} & =C_{l_{0}}(\alpha)+C_{l_{\beta}}(\alpha) \beta+C_{l_{p}}(\alpha) \frac{p b}{2 V}+C_{l_{r}}(\alpha) \frac{r b}{2 V}+C_{l_{\delta_{a}}}(\alpha) \delta_{a} \\
& +C_{l_{\delta_{r}}}(\alpha) \delta_{r} \\
C_{n} & =C_{n_{0}}(\alpha)+C_{n_{\beta}}(\alpha) \beta+C_{n_{p}}(\alpha) \frac{p b}{2 V}+C_{n_{r}}(\alpha) \frac{r b}{2 V}+C_{n_{\delta_{a}}}(\alpha) \delta_{a} \\
& +C_{n_{\delta_{r}}}(\alpha) \delta_{r}
\end{aligned}
$$

The attitude angles $\theta, \phi$ and $\psi$ for the aircraft model, mounted on the three-DOF gimbal, in trim conditions are related to the angle of attack $\alpha$ and sideslip $\beta$. For small roll and yaw angles, the following simplified relations can be used:

$$
\begin{array}{ll}
\phi \ll 1, & \psi \ll 1 \\
\alpha=\theta ; & \beta=\phi \sin \alpha+\psi
\end{array}
$$

The trim roll and yaw angles ( $\phi_{s}$ and $\psi_{s}$, respectively) are determined according to Eqs. (7-9) under zero rotation conditions $(p=r=0)$ :

$$
\begin{aligned}
\phi_{s} & =\frac{\Delta C_{l}(\alpha) C_{n_{\beta}}(\alpha)-\Delta C_{n}(\alpha) C_{l_{\beta}}(\alpha)}{k_{\phi}\left(C_{l_{\delta_{a}}}(\alpha) C_{n_{\beta}}(\alpha)-C_{n_{\delta_{a}}}(\alpha) C_{l_{\beta}}(\alpha)\right)} \\
\psi_{s} & =\frac{-\Delta C_{n}(\alpha)\left(C_{l_{\beta}}(\alpha) \sin \alpha+k_{\phi} C_{l_{\delta_{a}}}(\alpha)\right)+\Delta C_{l}(\alpha)\left(C_{n_{\beta}}(\alpha) \sin \alpha+k_{\phi} C_{n_{\delta_{a}}}(\alpha)\right)}{k_{\phi}\left(C_{l_{\delta_{a}}}(\alpha) C_{n_{\beta}}(\alpha)-C_{n_{\delta_{a}}}(\alpha) C_{l_{\beta}}(\alpha)\right)}
\end{aligned}
$$

wing-rock oscillations due to the local instability of the Dutch roll mode and nonlinear dependence of aerodynamic moments on sideslip and velocity roll rate. The local instability in the lateraldirectional modes can be stabilized using ailerons and rudder via feedback signals proportional to roll and yaw rates. The appearance of asymmetric aerodynamic moments will be indicated in the experiments by nonzero roll and yaw trim angles.

In the selected configuration, to allow the stabilization of equilibrium states and the elimination of the wing-rock regime, a simple controller was implemented using roll and yaw angles and angular rate feedback to the ailerons $\delta_{a}$ and rudder $\delta_{r}$. This is combined with the previously described longitudinal control using the aircraft-model elevator and the aerodynamic compensator:

$$
\begin{aligned}
\delta_{e} & =\delta_{e_{d}}+\frac{k_{\theta} s}{s+\omega} \theta+k_{q} q \\
\delta_{\mathrm{ec}} & =\delta_{\mathrm{ec}_{d}}+k_{q_{a}} q_{a} \\
\delta_{a} & =\delta_{a_{d}}+k_{\phi} \phi+k_{p} p \\
\delta_{r} & =\delta_{r_{d}}+\frac{k_{\psi} s}{s+\omega} \psi+k_{r} r
\end{aligned}
$$

in which

$$
\begin{aligned}
\Delta C_{l}(\alpha) & =C_{l_{0}}(\alpha)+C_{l_{\delta_{a}}}(\alpha) \delta_{a_{d}}+C_{l_{\delta_{r}}}(\alpha) \delta_{r_{d}} \\
\text { and } \quad \Delta C_{n}(\alpha) & =C_{n_{0}}(\alpha)+C_{n_{\delta_{a}}}(\alpha) \delta_{a_{d}}+C_{n_{\delta_{r}}}(\alpha) \delta_{r_{d}}
\end{aligned}
$$

In the case of no asymmetric aerodynamic moments, $C_{l_{0}}=C_{n_{0}}=0$, and with zero commanded control inputs, $\delta_{a_{d}}=\delta_{r_{d}}=0$, the aircraft-model roll and yaw angles [Eq. (10)] should be zero. Therefore, the trim angles $\phi_{s}$ and $\psi_{s}$ can serve as a measure of asymmetry in the aerodynamic moments, when $\delta_{a_{d}}=\delta_{r_{d}} \approx 0$.

Figures $8 \mathrm{a}$ and $8 \mathrm{~b}$ show the filtered values for quasi-static roll and yaw angles, $\phi_{s}(\bar{t})$ and $\psi_{s}(t)$, respectively, obtained using the maneuver rig with the aircraft pitch, roll, and yaw DOF released in addition to the arm pitch angle (which approximates aircraft heave). Tests in which the elevator deflection was slowly ramped up and down (with $\left|\dot{\delta}_{e_{d}}\right| \ll 1 \mathrm{deg} / \mathrm{s}$ ) were conducted. In these runs, command inputs to the controllers were $\delta_{a_{d}}=0$ and $\delta_{r_{d}}=0$, and the gain $k_{\psi}$ was set to zero. Figures $\underline{8 \mathrm{c}}$ and $\underline{8 \mathrm{~d}}$ show similar processes for the case when the rudder demand was nonzero, using $\delta_{a_{d}}=0$ and $\delta_{r_{d}}= \pm 10 \mathrm{deg}$. 


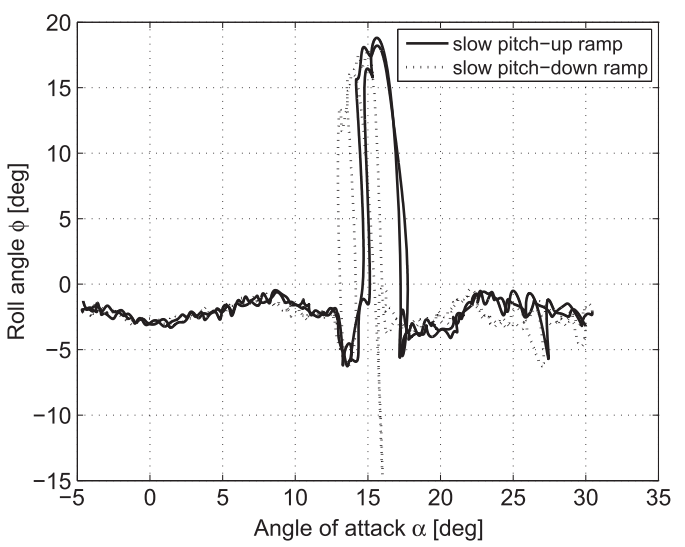

a) Dependence $\phi_{s}(\alpha)$ at $\delta_{r_{d}}=0$

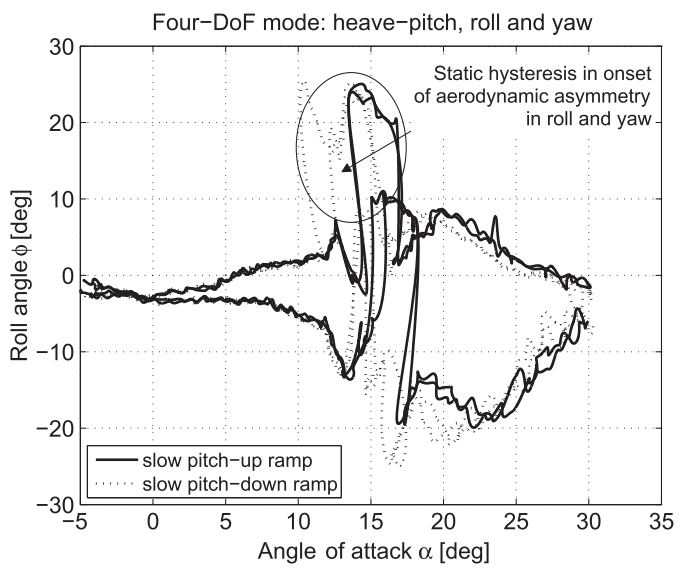

c) Dependence $\phi_{s}(\alpha)$ at $\delta_{r_{d}}= \pm 10^{\circ}$

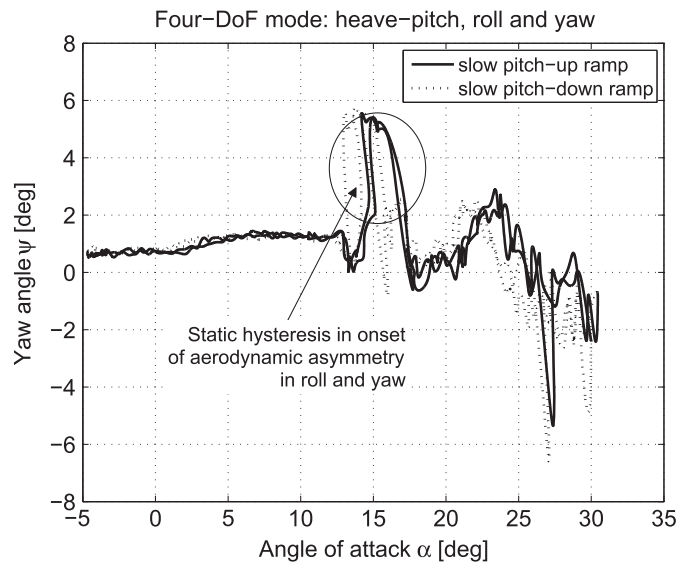

b) Dependence $\psi_{s}(\alpha)$ at $\delta_{r_{d}}=0$

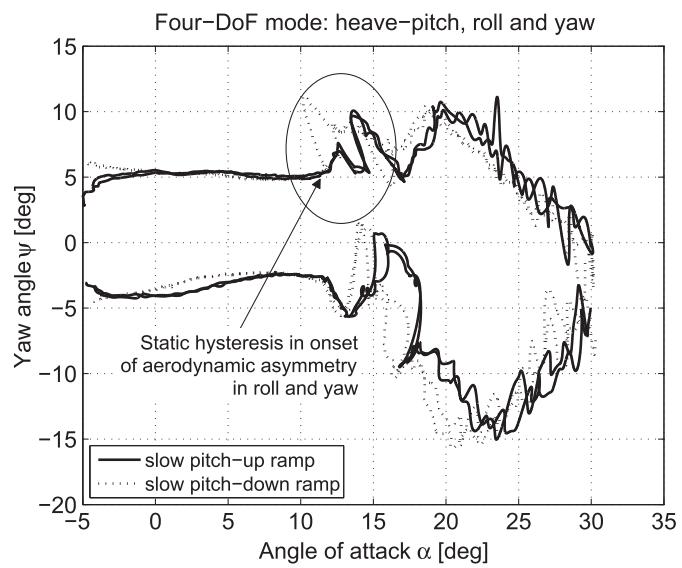

d) Dependence $\psi_{s}(\alpha)$ at $\delta_{r_{d}}= \pm 10^{\circ}$

Fig. 8 Stabilized trim angles $\phi_{s}$ and $\psi_{s}$ with model free in heave, pitch, roll, and yaw.

At low angles of attack and attached flow conditions $(\alpha=-5$ to $13 \mathrm{deg}$ ), the trim angles in roll and yaw shown in Figs. $8 \mathrm{a}$ and $8 \mathrm{~b}$ are quite small $\left(\phi_{s} \approx-2 \mathrm{deg}, \psi_{s} \approx 1 \mathrm{deg}\right)$. These asymmetric trim angles are probably associated with small imperfections in the aircraft-model geometry, which trigger a massive asymmetric flow separation and significantly increase the magnitudes of trim angles $\phi_{s}$ and $\psi_{s}$ in the range $\alpha=14$ to $17.5 \mathrm{deg}$. At higher angles of attack, $\alpha>17 \mathrm{deg}$, the level of asymmetric aerodynamic moments reduces to that at low incidences. With further increase in angle of attack, $\alpha>20 \mathrm{deg}$, asymmetry in the yaw moment becomes more prominent. Therefore, the experimental results shown in Fig. 8 also indicate the existence of the hysteretic dependencies in the asymmetric aerodynamic loads.

The aerodynamic asymmetry detected in the tests on the maneuver rig with heave, pitch, roll, and yaw DOF is located in the range of angle of attack $\alpha=14$ to $17.5 \mathrm{deg}$, which matches well with the location of the intermediate equilibrium branches in the presumed bifurcation diagrams shown in Figs. $\underline{4}$ and $7 \mathrm{~b}$. The onset of this massive aerodynamic asymmetry in the rolling $C_{l_{0}}$ and yawing $C_{n_{0}}$ moments has a distinct hysteretic dependence in the pitch-up (solid lines) and pitch-down (dashed lines in Fig. 7b) ramp motions with an angle of attack shift of $\Delta \alpha \approx \pm 2 \mathrm{deg}$. This corresponds closely with the earlier test results obtained with the rig configured as pitch only and with heave-and-pitch DOF. These observations clearly show that asymmetric flow separation phenomena are an important feature shaping the aerodynamic loads in the stall region of the tested Hawk model.

\section{Conclusions}

This paper presented the results of an experimental study of stall aerodynamics via stabilization of unstable trim states of an approximate copy of a one-sixteenth-scale Hawk aircraft model. The tests have been conducted using the updated University of Bristol multi-DOF maneuver rig in a low-speed wind tunnel. The aircraft model was attached via a three-DOF gimbal to an arm, which itself is free to move in up to three DOF about a point set back from the model, such that the approximate vertical and horizontal translational motions of the model can be simulated. In addition to the experimental results, this paper presented the improvements in hardware and communication systems implemented in the experimental rig.

The experimental rig has a number of advantages compared with traditional static and dynamic testing apparatus. The rig can be configured to allow the Hawk model to be free in pitch, plunge, roll, and yaw in different combinations, and allows a significant reduction in aeroelastic coupling between an aircraft model and its support system compared with traditional static and dynamic testing. Note that, in traditional testing, when the aircraft model is firmly fixed on a sting with a strain gauge balance, flow instability and aerodynamic buffet in the stall region excite structural vibrations of the support sting; this has a considerable intrusive effect on the separated flow structure, affecting measured aerodynamic loads. Possible hysteretic dependencies in static conditions may be significantly transformed and effectively linearized, so that aerodynamics loads show only single discontinuous or steep changes. The structural dynamic effects on the maneuver rig as well as on traditional model-fixed testing facilities require further investigation.

The rig compensator and the Hawk model have actuated aerodynamic control surfaces, which are used for trimming the aircraft model and for stabilization of its unstable equilibria. These control surfaces are operated in attached flow conditions and are regarded as linear control effectors. Their influence on the flow around the Hawk model is assumed in this work to be small. 
The dynamic rig with active stabilization of unstable modes of the freely moving model allows deeper insight into stall aerodynamics. The obtained experimental results clearly show the existence of hysteresis phenomena with abrupt jumps between different branches in the dependencies of the normal aerodynamic force observed in static stall conditions. The qualitative bifurcation analysis of the measured aerodynamic dependencies at zero sideslip indicates that the intermediate branch of the aerodynamic hysteresis, in some range of angles of attack, is associated with the onset of asymmetric rolling and yawing aerodynamic moments due to an asymmetric separated flow structure. The observed static hysteresis phenomena prove that the proposed concept of testing is opening new opportunities in experimental testing of stall aerodynamics, helping to identify possible causes of the onset of LCOs.

The measured static aerodynamic hysteresis demonstrates the expanded capabilities of the updated and actively controlled windtunnel maneuver rig with improved hardware and communication software. In addition to facilitating physical simulations of the aircraft-model dynamics at stall conditions, it serves as a minimally intrusive system-minimizing the coupling of support system structural dynamics with the unsteady flow dynamics-for investigation of separated flow conditions by experimental continuation of stabilized aircraft-model trim states. The advantages delivered by this actively controlled testing approach make it a valuable complement to traditional wind-tunnel test techniques.

The findings in this paper suggest promising prospects for the multi-DOF testing methodology. Thorough comparisons with other experimental methods are needed, and further computational and experimental studies are required to gain a comprehensive understanding of unsteady nonlinear aerodynamic phenomena in the stall region.
Table A2 Mass and c.g. position of combined rig/Hawk model/compensator

\begin{tabular}{lc}
\hline \hline Mass $M$ & $9.53 \mathrm{~kg}$ \\
Position of c.g. ahead of arm gimbal center $X_{\text {c.g. }}$ & $0.109 \mathrm{~m}$ \\
Position of c.g. below arm gimbal center $Z_{\text {c.g. }}$ & $0.0417 \mathrm{~m}$ \\
\hline
\end{tabular}

Table A3 Controller constant

\begin{tabular}{lcccc}
\hline \hline Quantity & Unit & Eq. (1) & Eq. (6) & Eq. (7) \\
\hline$k_{q}$ & $\mathrm{~s}$ & 0.28 & 0.28 & 0.28 \\
$k_{\theta}$ & -- & 0.60 & 0.60 & 0.60 \\
$\omega$ & $\mathrm{rad} / \mathrm{s}$ & 0.20 & 0.20 & 0.20 \\
$k_{q_{a}}$ & $\mathrm{~s}$ & -- & 1.0 & 1.0 \\
$k_{\phi}$ & -- & -- & -- & 0.40 \\
$k_{p}$ & $\mathrm{~s}$ & -- & -- & 0.10 \\
$k_{\psi}$ & -- & -- & -- & 2.0 \\
$k_{r}$ & $\mathrm{~s}$ & -- & -- & 1.0 \\
\hline \hline
\end{tabular}

\section{Acknowledgments}

This work was supported by a National Natural Science Foundation of China grant, number 11402115. Zheng Gong was supported by both the China Scholarship Council fellowship for one year at the University of Bristol and a Fundamental Research Funds for the Central Universities of China grant, number NS2017006. Sergio Araujo-Estrada's research was supported by the Science and Technology National Council (Consejo Nacional de Ciencia y Tecnología, Mexico), studentship number 215262. Simon Neild is supported by an Engineering and Physical Sciences Research Council fellowship, EP/K005375/1. Bai Yalei from Nanjing University of Aeronautics and Astronautics, Nanjing, People's

Table A1 Hawk model, compensator, and rig characteristics

\begin{tabular}{lccc}
\hline \hline Quantity & Aircraft model & Compensator & Rig arm \\
\hline Mass, $\mathrm{kg}$ & 1.97 & 3.91 & 3.65 \\
Position of c.g. ahead of arm gimbal center, $\theta_{a}=0, \mathrm{~m}$ & 0.80 & -0.38 & 0.262 \\
Position of c.g. below arm gimbal center, $\theta_{a}=0, \mathrm{~m}$ & 0 & 0 & 0.109 \\
Reference area, $\mathrm{m}^{2}$ & 0.0796 & 0.125 & \\
Mean aerodynamic chord, $\mathrm{m}$ & 0.143 & 0.181 & \\
$1 / 4$-chord sweep, deg & 21.7 & 7.12 & \\
Span, $\mathrm{m}$ & 0.594 & 0.700 & \\
Moment of inertia about $x$ body axis, $\mathrm{kg} \cdot \mathrm{m}^{2}$ & $8.08 \times 10^{-3}$ & & \\
Moment of inertia about $y$ body axis, $\mathrm{kg} \cdot \mathrm{m}^{2}$ & $4.76 \times 10^{-2}$ & & \\
Moment of inertia about $x$ body axis, $\mathrm{kg} \cdot \mathrm{m}^{2}$ & $6.12 \times 10^{-2}$ & \\
\hline \hline
\end{tabular}
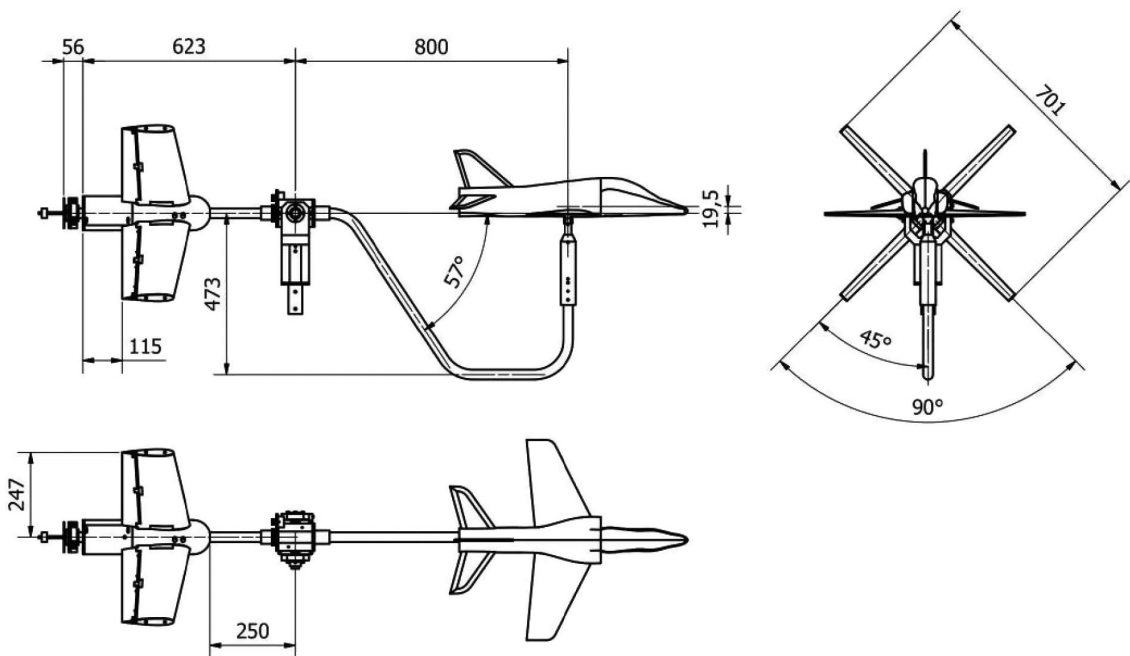

Fig. A1 Rig dimensions. 
Table A4 Data for $\delta_{e_{d}}$ as a function of trimmed $\alpha$

\begin{tabular}{lcccccccc}
\hline \hline$\alpha, \mathrm{deg}$ & -8.0991 & -6.3681 & -4.3233 & -2.9083 & -1.4130 & -0.075879 & 1.8904 & 4.0136 \\
$\delta_{e_{d}}, \mathrm{deg}$ & 8.6826 & 7.0658 & 5.8084 & 5.4491 & 3.8323 & 2.9341 & 1.6767 & 0.59880 \\
$\alpha, \mathrm{deg}$ & 5.9801 & 7.9466 & 9.5986 & 11.879 & 12.824 & 14.634 & 16.700 & 17.000 \\
$\delta_{e_{d}}, \mathrm{deg}$ & -0.83832 & -2.2755 & -3.5329 & -4.9701 & -6.4072 & -7.6647 & -11.000 & -13.144 \\
$\alpha, \operatorname{deg}$ & 18.652 & 19.912 & 20.938 & 22.276 & 23.222 & 24.561 & 25.820 & - \\
$\delta_{e_{d}}, \mathrm{deg}$ & -15.928 & -18.084 & -20.778 & -22.755 & -24.910 & -27.156 & -29.132 & - \\
\hline \hline
\end{tabular}

Republic of China, provided the computational fluid dynamics simulation data for the Hawk model at low angles of attack, which were used for control law design.

\section{References}

18

[1] Anon., "Cooperative Programme on Dynamic Wind Tunnel Experiments for Manoeuvring Aircraft," AGARD- AR-305, 1996.

[2] Chambers, J. R., "Modeling Flight: The Role of Dynamically Scaled Free-Flight Models in Support of NASA's Aerospace Programs," NASA SP 2009-575, 2009.

[3] Owens, D. B., Brandon, J. M., Croom, M. A., Fremaux, C. M., Heim, E. H., and Vicroy, D. D., "Overview of Dynamic Test Techniques for Flight Dynamics Research at NASA LaRC," 25th AIAA Aerodynamic Measurement Technology and Ground Testing Conference, AIAA Paper 2006-3146, 2006. doi: $10.2514 / 6.2006-3146$

[4] Huang, M., and Wang, Z.-W., "A Review of Wind Tunnel Based Virtual Flight Testing Techniques for Evaluation of Flight Control Systems," International Journal of Aerospace Engineering, 2015, Paper 672423. doi:10.1155/2015/672423

[5] Ignatyev, D. I., Sidoryuk, M. E., Kolinko, K. A., and Khrabrov, A. N., "Dynamic Rig for Validation of Control Algorithms at High Angles of Attack," Journal of Aircraft, Vol. 54, No. 5, 2017, pp. 1760-1771. doi: $10.2514 / 1 . C 034167$

[6] Li, H., Zhao, Z.-L., and Fan, Z.-L., "Simulation Method for Wind Tunnel Based Virtual Flight Testing," International Journal of Modern Physics: Conference Series, Vol. 19, 2012, pp. 381-389. doi:10.1142/S2010194512008975

[7] Muse, J. A., Kutayy, A., and Calise, A. J., "A Novel Force Control Traverse for Simulating UAV Flight in a Wind Tunnel," AIAA Atmospheric Flight Mechanics Conference and Exhibit, AIAA Paper 2008-6714, 2008. doi: $10.2514 / 6.2008-6714$

[8] Chen, J., Shen, X., Tu, F., and Qureshi, E. M., "Experimental Research on an Active Sting Damper in a Low Speed Acoustic Wind Tunnel," Shock and Vibration, Vol. 2014, 2014, Paper 524351. doi: $10.1155 / 2014 / 524351$

[9] Balakrishna, S., Houlden, H., Butler, D. H., and White, R., "Development of a Wind Tunnel Active Vibration Reduction System," Collection of Technical Papers-45th AIAA Aerospace Sciences Meeting and Exhibit, Vol. 17, AIAA, Reston, VA, Jan. 2007, pp. 1166111672 .
[10] Ye, J. Z., Jiang, Y. W., Zhou, N. Z., Song, B. F., and He, Z. J., "The Aeroelastic Effects on the Scatter Phenomenon of Wind-Tunnel Data," Science China Technological Sciences, Vol. 56, No. 2, Feb. 2013, pp. $405-415$. doi:10.1007/s11431-012-5100-6

[11] Pattinson, J., "Development and Evaluation of a Wind Tunnel Manoeuvre Rig," Ph.D. Thesis, Univ. of Bristol, Bristol, England, U.K., 2010.

[12] Pattinson, J., Lowenberg, M. H., and Goman, M. G., "Multi-Degree-ofFreedom Wind-Tunnel Maneuver Rig for Dynamic Simulation and Aerodynamic Model Identification," Journal of Aircraft, Vol. 50, No. 2, 2013, pp. 551-566. doi:10.2514/1.C031924

[13] Pattinson, J., Lowenberg, M. H., and Goman, M. G., "Investigation of Poststall Pitch Oscillations of an Aircraft Wind-Tunnel Model," Journal of Aircraft, Vol. 50, No. 6, 2013, pp. 1843-1855. doi:10.2514/1.C032184

[14] Araujo-Estrada, S. A., Lowenberg, M., Neild, S., and Goman, M., "Evaluation of Aircraft Model Upset Behaviour Using Wind Tunnel Manoeuvre Rig," AIAA Atmospheric Flight Mechanics Conference, AIAA Paper 2015-0750, 2015. doi:10.2514/6.2015-0750

[15] Araujo-Estrada, S. A., Gong, Z., Lowenberg, M., Neild, S., and Goman, M., "Wind Tunnel Manoeuvre Rig: A Multi-DOF and HAOA Testing Platform for Model Aircraft," AIAA Science and Technology Forum and Exposition, AIAA Paper 2016-2119, 2016. doi:10.2514/6.2016-2119

[16] digi.com, XBee Wi-Fi, http://www.digi.com/products/xbee-rf-solutions/ modules/xbee-wi-fi [retrieved 14 Dec. 2015].

[17] Mills, D. L., "Network Time Protocol Version 4 Reference and Implementation Guide," Network Time Protocol Working Group, Univ. of Delaware, Rept. 06-6-1, 2006.

[18] Carnduff, S., Erbsloeh, S., Cooke, A., and Cook, M., "Development of a Low Cost Dynamic Wind Tunnel Facility Utilizing MEMS Inertial Sensors," 46th AIAA Aerospace Sciences Meeting and Exhibit, AIAA Paper 2008-0196, 2008.

[19] Versteeg, H. K., and Malalasekera, W., An Introduction to Computational Fluid Dynamics: The Finite Volume Method, 2nd ed., Pearson, 2007.

[20] Anon., ANSYS Fluent User's Guide, Release 16.2, ANSYS, Canonsburg, PA, 2015. 